\title{
COSTE-EFECTIVIDAD DE LA TERAPIA FOTODINÁMICA EN LA DEGENERACIÓN MACULAR ASOCIADA A LA EDAD
}

\section{COST-EFFECTIVENESS OF PHOTODYNAMIC THERAPY IN AGE-RELATED MACULAR DEGENERATION}

\author{
MUSLERA E ${ }^{1}$, NATAL $C^{2}$
}

\begin{abstract}
RESUMEN
Objetivo: Estimar el coste para el servicio público de salud de la mejora o el mantenimiento de la agudeza visual en pacientes con degeneración macular asociada a la edad mediante la aplicación de la terapia fotodinámica con verteporfina.

Método: Estudio coste efectividad desde la perspectiva de un servicio de salud teniendo en cuenta la alternativa no tratar. La medida de eficacia es la ganancia o mantenimiento de la agudeza visual y la sensibilidad al contraste. Los costes directos ligados al proceso de la intervención son estimados a partir de la contabilidad analítica. El coste ajustado por calidad, se estima con valores procedentes de otros estudios.

Resultados: El coste por año ganado de mantenimiento de la agudeza visual, en un horizonte de dos años es de $36.530 €$ para mujeres y $34.804 €$ para hombres. Si el horizonte fuera la esperanza de vida en Asturias, el coste disminuye a $4.298 €$ y $5.354 €$ respectivamente. El coste por año ganado de mantenimiento de agudeza visual ajustado por calidad es,
\end{abstract}

\begin{abstract}
Objective: The aim of this study was to estimate the public health service cost of visual acuity improvement or maintenance with photodynamic therapy in patients with age-related macular degeneration (ARMD). This illness is the most frequent cause of blindness in elderly patients in western countries.

Methods: A cost-effectiveness analysis was carried out to compare photodynamic therapy versus no treatment. The analysis point of view was that of the health service. The improvement or maintenance of visual acuity and contrast sensitivity were considered efficacy results. Direct costs were estimated by means of cost accountancy. Quality adjusted costs per visual acuity life year gained (QACVAG) were calculated through utility values from other studies.

Results: The cost per year of maintenance of visual acuity in a two-year period was $36,530 €$ for women and $34,804 €$ for men. If this cost was estimated for life expectancy in Asturias, it would be reduced to 4,298 € for women and 5,354 € for men.
\end{abstract}

Recibido: 23/6/05. Aceptado: 19/4/06.

Unidad de Análisis y Programas. Servicio de Salud del Principado de Asturias. España.

1 Licenciada en Ciencias Económicas.

2 Licenciada en Medicina.

Comunicación presentada parcialmente en XXIV Jornadas de Economía de la Salud (El Escorial 2001) y en el XXI Congreso de la Sociedad Española de Calidad Asistencia (Oviedo 2003).

Correspondencia:

Elvira Muslera Canclini

Unidad de Análisis y Programas

Servicio de Salud del Principado de Asturias

Plaza del Carbayón, 1 y 2, 3. ${ }^{a}$ planta

33001 Oviedo

España

E-mail: elvira.muslera@sespa.princast.es 
en horizonte de dos años, de $66.931 €$ para mujeres y $70.249 €$ para los hombres.

Conclusión: Los estudios coste-efectividad facilitan decidir sobre la pertinencia de la financiación pública. Algunos autores sugieren incluir sólo aquellas intervenciones que no superen los 30.000 $€$ por año ganado de mantenimiento de agudeza visual ajustado por calidad y en este estudio se sobrepasa holgadamente este valor. Es recomendable una selección más rigurosa de los pacientes, tanto por criterios diagnósticos como por características individuales en relación con su autonomía, lo que permitiría mejorar los resultados de esta intervención.

Palabras claves: Coste-efectividad, terapia fotodinámica, degeneración macular asociada a la edad.
If costs of the QACVAG, in a two-year period, were considered, photodynamic therapy would cost $66,931 €$ for women and 70,249 € for men.

Conclusion: This cost-effectiveness analysis allows decisions to be made about public financing. Some research in our country suggests that public health financing should be provided for interventions whose cost-effectiveness is less than $30,000 €$ of CVAQA. The treatment evaluated here far exceeds this value. It is recommended that the use of more restrictive patient selection, incorporating diagnostic criteria and patient autonomy indicators, could improve the results of this intervention (Arch Soc Esp Oftalmol 2006; 81: 199-204).

Key words: Cost-effectiveness, photodynamic therapy, age-related macular degeneration.

\section{INTRODUCCIÓN}

La degeneración macular asociada a la edad (DMAE) es una de las principales causas de ceguera en nuestro medio en mayores de 50 años, representando entre el $50 \%$ y el $77 \%$ según las diferentes fuentes de información (1-3).

La prevalencia de esta enfermedad varía según los criterios y los métodos diagnósticos utilizados; el estudio realizado por el Eye Diseases Prevalence Research Group estima una prevalencia de DMAE para todas las edades de 1,47\% (4). La prevalencia aumenta con la edad, encontrándose en 2,3\% para mayores de 70 años (5). La forma exudativa tiene una prevalencia de $0,88 \%$. Por edades, la prevalencia es del 0,17\% para el grupo de 55-64 años, 0,54\% para el de 65-74 años, 2,52\% entre los 75 y 84 años y $5,76 \%$ para los mayores de 85 años. El $36 \%$ de éstas se presentan como lesiones de neovascularización coroidea (NVC) subfoveales (6), clásicas y potencialmente tratables con terapia fotodinámica (TF) con verteporfina. Extrapolando estos datos a Asturias se estima que hay actualmente 1.282 personas afectadas de DMAE susceptibles de tratamiento con TF. Un número de ellas tendrá afectación de ambos ojos. Por grupos de edad la mayor frecuencia se encuentra entre los 75 y 84 años, con el $45 \%$ de los afectados. Los mayores de 84 años suponen el $33 \%$.

Además de la TF se han intentado diferentes tratamientos para la DMAE como la fotocoagulación con láser, la radioterapia a bajas dosis, la cirugía y el uso de agentes angiogénicos y angiostáticos y la termoterapia transpupilar.

La fotocoagulación con láser tiene buenos resultados en cuanto a la conservación de la agudeza visual y preservación de la sensibilidad al contraste en pacientes con NVC secundaria a DMAE exudativa, pero debido a que no actúa selectivamente sobre el tejido patológico sólo está indicada en lesiones extrafoveales y yuxtafoveales (7). La radioterapia a bajas dosis ha sido estudiada sin obtener todavía resultados concluyentes $(8,9)$.

Actualmente existen expectativas con relación a los resultados de la termoterapia transpupilar que podría ser la alternativa a la TF (10).

La Agència d'Avaluació de Tecnologia i Recerca Mèdiques establece las siguientes recomendaciones sobre TF en el tratamiento de la DMAE: tratar sólo a pacientes con evidencia de un patrón clásico subfoveal de NVC igual o superior al 50\% del total de la superficie de la lesión; centralizar el tratamiento y limitar la oferta pública a centros hospitalarios con amplia experiencia en patología macular; mantener esta centralización dos o tres años como mínimo y realizar una valoración anual, estrechamente ligada a su financiación (11).

El objetivo de este estudio es estimar el coste de la mejora o el mantenimiento de la agudeza visual en pacientes con NVC subfoveal predominantemente clásica debida a DMAE mediante la aplicación de la TF con verteporfina. 


\section{SUJETOS, MATERIAL Y MÉTODO}

Se realiza un estudio coste-efectividad desde la perspectiva de un servicio de salud, teniendo en cuenta la alternativa no tratar.

\section{Medida de la eficacia}

La eficacia de la TF ha sido medida en dos ensayos clínicos: Verteporfin in Photodynamic Therapy (VIP) y Treatment of Age-Related Macular Degeneration with Photodynamic Therapy (TAP) $(12,13)$, ambos realizados en centros de Europa y los Estados Unidos de América. La principal diferencia entre ambos se encuentra en los criterios de selección de pacientes, siendo éstos más restrictivos para el estudio realizado por el TAP (13). Mientras éste incluye solamente pacientes mayores de 50 años, con agudeza visual comprendida entre 20/40 y 20/200 y neovascularización coroidea clásica o predominantemente clásica causada por DMAE, el estudio VIP incluye pacientes con mejor agudeza visual, más jóvenes y con neovascularización coroidea de diferentes causas (13).

Estos ensayos utilizan tres medidas de resultado: la agudeza visual (cuya variable principal es la tasa de respuesta, definida como la proporción de pacientes que perdieron menos de 3 líneas de agudeza visual con relación al valor basal), la sensibilidad al contraste y la clasificación semicuantitativa angiográfica de las lesiones de retina.

La agudeza visual se evaluó utilizando el equivalente de Snellen en un Test de Agudeza Visual a Distancia con un optotipo Ligthhouse siguiendo una refracción estandarizada. La escala de clasificación es: a) Mejoría de la visión, aumento de una o más líneas de agudeza visual; b) visión estable, pérdida de menos de 3 líneas o sin cambios; c) pérdida moderada, entre 3 y 5 líneas y d) pérdida grave, 6 o más líneas.
La sensibilidad al contraste es evaluada en el ensayo TAP, resultando que los pacientes bajo tratamiento conservaron, a los dos años, 6 letras $(\mathrm{p}<0,001)(14)$.

En el presente estudio se han utilizado los datos de eficacia resultantes del ensayo clínico TAP (13), ya que la selección de los pacientes se asemejaba a nuestro grupo de pacientes: edad avanzada, neovascularización coroidea clásica o predominantemente clásica secundaria a DMAE. Del ensayo se desprende que, a los dos años del tratamiento, un 15,3\% $(\mathrm{p}<0,001)$ más de pacientes pierden menos de 3 líneas, es decir que mantienen una visión estable, en comparación con los que se les administró placebo.

La TF no presenta resultados de curación, por esta razón sus beneficios consisten en evitar el empeoramiento de la visión o frenar la ceguera. El beneficio esperado se expresa como la mayor proporción de personas que conservan o mejoran la agudeza visual, el 15,3\% en el estudio TAP, en comparación con las personas que no reciben tratamiento.

La medida de resultado intermedio es la fibrosis, que constituye motivo de alta. Se puede decir, sin embargo, que estos dos resultados son complementarios porque, aunque se detecte mejoría, no puede suspenderse el tratamiento hasta que se compruebe la fibrosis, por medio de la angiografía. Aunque también es posible que se detecte fibrosis con empeoramiento de la visión.

Como medida adicional de resultado se ha considerado la ganancia de agudeza visual ajustada por calidad, para su cálculo se recurre a las medidas de utilidad estimadas por Brown et al (15) que se presentan en la tabla I.

Para este estudio se seleccionan los primeros 50 pacientes atendidos, que son los han cumplido un ciclo completo de tratamiento. En siete casos son tratados los dos ojos. Los criterios de selección de pacientes se evalúan regularmente mediante audits, cumpliéndose los criterios propuestos (11) en todos los casos (16).

Tabla I. Medida de utilidad según agudeza visual (15)

\begin{tabular}{lccc}
\hline Estado & Agudeza visual & Utilidad & IC 95\% \\
\hline Normalidad & $20 / 20-20 / 25$ & 0,89 & $0,82-0,96$ \\
Intermedio 1 & $20 / 30-20 / 50$ & 0,81 & $0,73-0,89$ \\
Intermedio 2 & $20 / 60-20 / 100$ & 0,57 & $0,47-0,67$ \\
Ceguera legal & $20 / 200-20 / 400$ & 0,52 & $0,38-0,66$ \\
Ceguera & Contar dedos hasta percepción de la luz & 0,40 & $0,29-0,50$ \\
\hline \hline
\end{tabular}




\section{Medida de la utilización de recursos}

El itinerario seguido por las personas atendidas comienza con una consulta en el servicio de oftalmología del hospital de referencia regional para confirmar el diagnóstico. En caso afirmativo es derivado al hospital de área donde se atiende a los pacientes de toda la comunidad autónoma. En éste se realiza, en el mismo día, el test de agudeza visual, la angiografía y el tratamiento con la verteporfina. Finalmente se concierta una cita de revisión a los tres meses. Estas sesiones se repiten como máximo, ocho veces.

Es posible ampliar la perspectiva de estudio incorporando el supuesto de que la agudeza visual permanece durante la esperanza de vida y agregando información respecto a la calidad de vida de las personas afectadas.

Para el primer supuesto se utilizan las estimaciones para Asturias de esperanza de vida en hombres a los 70-74 (tramo de edad con la que entran en tratamiento), que es de 13 años, y para las mujeres, en ese mismo tramo, que se eleva a 17 años.

También se realiza una estimación del coste ajustado por calidad de mantenimiento de la agudeza visual, que se calcula aplicando los índices de utilidad del estudio de Sharma a los años ganados de agudeza visual en nuestra población (17), ya que nuestro estudio es retrospectivo y no es posible aplicar ningún índice de calidad de vida.

Para el cálculo de costes se utiliza la contabilidad analítica del hospital de área. En la estimación se consideran los costes directos ligados al proceso de la intervención desde que la persona es diagnosticada hasta el momento del alta por fibrosis o a los dos años de iniciado el tratamiento.

\section{RESULTADOS}

Del total de tratamientos aplicados el 63,15\% (36 ojos) son alta por remisión de la lesión (fibrosis). Sólo en un caso hay alta por fracaso del tratamiento en la 6. ${ }^{\text {a }}$ sesión y se pierden 16 de pacientes en dos años.

El número medio de sesiones recibidas es de 5,08 sesiones por paciente. Se produce fibrosis en el $70 \%$ de los pacientes que siguen un ciclo completo. Los abandonos se acumulan en las últimas sesiones.

El coste más importante es el medicamento, verteporfina, que representa el $65 \%$ del coste de cada sesión (tabla II), seguido de la amortización del equipamiento, el láser oftálmico fotocoagulador, con el $26 \%$.
El coste total (coste de todas las sesiones realizadas a los pacientes en el período) se eleva a $549.611 €$. El coste por paciente tratado es de $10.729 €$ y el coste por paciente «con éxito» - aquellos que mejoran o mantienen la agudeza visual debido a la aplicación de la TF, diferencia entre alternativa no tratar y el tratamiento- se eleva a $71.525 €$ (tabla III).

El coste marginal (coste de cada sesión adicional) en la cuarta sesión alcanza el valor mínimo de 6.993 $€$ y en la quinta se eleva a $28.050 €$, siendo éste el valor más alto. Esta situación es predecible, ya que el número de casos favorables acumulados aumenta cada vez en menor proporción, siendo sólo uno más, entre la sesión séptima y octava.

En el grupo estudiado sólo el $12 \%$ presenta una leve mejoría, el $70 \%$ mantiene la agudeza visual estable y el $18 \%$ restante empeora. La edad media de las mujeres es de 71,2 años y la de los hombres es de 71,8 años.

Teniendo en cuenta los datos de eficacia del ensayo clínico TAP sólo un 15\% más de personas some-

Tabla II. Costes por sesión en el hospital del estudio

\begin{tabular}{lrr}
\hline Costes por concepto & Euros & $\%$ \\
\hline 1. Coste de personal & 39,4 & 2 \\
2. Coste de material & $1.301,6$ & 68 \\
Material (inyección de verteporfina ) & $1.252,2$ & \\
Material necesario para la Angiografía & 49,4 & \\
3. Coste gestión del almacén & 39,4 & 2 \\
4. Coste de los equipos & 505,0 & 26 \\
Láser oftálmico & 251,7 & \\
Bomba de Infusión & 253,3 & \\
5. Costes generales & 30,2 & 2 \\
Mantenimiento, limpieza, agua, & 30,2 & \\
electricidad, seguridad, papeles & & \\
Coste total de la sesión & $\mathbf{1 . 9 1 5 , 7}$ & $\mathbf{1 0 0 \%}$ \\
\hline \hline
\end{tabular}

Tabla III. Coste por paciente y por año ganado de agudeza visual en el Principado de Asturias

\begin{tabular}{lc}
\hline Costes & Importe \\
\hline Coste por paciente & $10.729 €$ \\
Coste por paciente que mantiene & $71.525 €$ \\
$\quad$ o mejora la visión & \\
Coste por año de mantenimiento de la & \\
$\quad$ agudeza visual (2 años) & $34.804 €$ \\
• Hombres & $36.530 €$ \\
• Mujeres & \\
Coste por año de mantenimiento de la & \\
$\quad$ agudeza visual (suponiendo estabilidad & \\
$\quad$ durante la esperanza de vida) & $5.354 €$ \\
• Hombres (13 años) & $4.298 €$ \\
• Mujeres (17 años) & \\
\hline \hline
\end{tabular}


tidas a la TF consiguen una visión estable (pérdida de menos de tres líneas) con respecto a los que se les administra placebo. Aplicando esta medida de efectividad a nuestros datos, se obtienen los valores de coste que se presentan en la tabla II. El coste por año ganado de mantenimiento de la agudeza visual, en un horizonte de dos años - tiempo sobre el que se dispone de información - es de $34.804 €$ para hombres y $36.530 €$ para mujeres. La tasa de descuento considerada es del 2,5\%.

En un escenario de dos años, la estimación del coste por año ganado de agudeza visual ajustado por calidad, es de $66.931 €$ para las mujeres y $70.249 €$ para los hombres. Esta estimación se presenta en la tabla IV.

\section{DISCUSIÓN}

Aunque con algunos interrogantes, sujetos a la espera de los resultados de los ensayos que aún se están realizando (18), la TF es, en estos momentos, la terapéutica de elección en la DMAE exudativa. La alternativa es la de no hacer nada, ya que otras opciones terapéuticas o están en fase experimental o han resultado tener una eficacia despreciable.

Desde el punto de vista de los servicios de salud es preciso determinar la relación coste-efectividad de la TF y, a la luz de los resultados, establecer si está justificada su financiación con fondos públicos.

En el presente estudio, el coste para el servicio de salud por año de conservación de la agudeza visual en las personas afectadas por DMAE, aplicando la TF, se aproxima a los $36.000 €$. Y los beneficios que aporta consisten en mantener o mejorar ligeramente una agudeza visual. Por esta razón es importantísimo que las personas que se someten a tratamiento presenten una agudeza visual buena, distante de la situación actual que se encuentra en el límite de la ceguera legal.

Tabla IV. Coste por año ganado de agudeza visual ajustado por calidad

\begin{tabular}{lc}
\hline Conceptos & Importe \\
\hline $\begin{array}{l}\text { Coste por año de mantenimiento } \\
\text { de la agudeza visual (2 años) }\end{array}$ \\
$\begin{array}{l}\text { • Hombres } \\
\text { • Mujeres }\end{array}$ \\
Coste por año de mantenimiento de la & $70.249 €$ \\
agudeza visual (durante la esperanza de vida) & \\
- Hombres (13 años) & $9.743 € €$ \\
- Mujeres (17 años) & $7.794 €$ \\
\hline \hline
\end{tabular}

Si se supone que los resultados se mantienen durante la esperanza de vida, los costes se reducen sustancialmente: $5.354 €$ para los hombres y $4.298 €$ para las mujeres (tabla III). Este escenario también lo presentan Sharma et al. que consideran una esperanza de vida de 11 años, sin hacer distinción por sexo (17).

Para justificar el grado de prioridad que puede otorgarse a la TF dentro de la cartera de servicios pública, se dispone del criterio establecido por el estudio de Sacristán et al. que consideran costeefectivas, en nuestro país, las intervenciones cuyo valor por año de vida ganado ajustado por calidad no exceda los $30.000 €$ (19). Aunque la medida de resultado en salud es algo diferente en este estudio - año de mantenimiento de la agudeza visual ajustado por calidad - debe tenerse en cuenta esta referencia en la toma de decisiones, ya que el coste para ese resultado se eleva a $68.000 €$. Con un análisis similar, Sharma et al (17), valoran que esta intervención es moderadamente coste-efectiva para pacientes con una agudeza inicial de 20/40 y costeinefectiva para pacientes con 20/200 (ésta es la agudeza visual que presentan la mayoría de nuestros pacientes, sólo el $28 \%$ de los mismos se encuentran por encima de ese valor). A la misma conclusión llegan Hopley et al (20) tres años más tarde.

Si se estima que los beneficios se mantienen hasta que las personas alcanzan su esperanza de vida los resultados son muy diferentes, descendiendo a $5.354 €$ para los hombres y $4.298 €$ para las mujeres. El horizonte temporal de la esperanza de vida para el análisis de la TF ha sido utilizado por otros autores (18), pero tiene importantes limitaciones, ya que no hay, aún, evidencia documentada que acredite que los beneficios se mantienen a tan largo plazo. Sería más adecuado que los beneficios futuros estuvieran relacionados con evaluaciones más globales de las personas enfermas, como por ejemplo, los índices funcionales, ya que, el mantenimiento de la visión en personas ancianas autónomas tiene mayor valor que en caso de las que no lo son. De esta forma sería posible construir escenarios de costes para beneficios distintos.

Las limitaciones del estudio se encuentran en primer lugar en el carácter retrospectivo del mismo, que impide la utilización de cuestionarios de calidad de vida y un mayor control de la aplicación de la TF en ambos ojos. Otras limitaciones están en relación con el tamaño de la muestra de pacientes estudiada, debido a que se trata de una enfermedad de baja prevalencia en población general, con una 
intervención de larga duración y de reciente introducción en la práctica clínica habitual. El número de pacientes que finalizan el tratamiento es limitado y no permite otros análisis. No obstante, estas limitaciones no invalidan los resultados presentados, que deben considerarse como una herramienta de apoyo a las decisiones para la gestión de los recursos de los servicios públicos de salud.

Las recomendaciones que surgen del estudio son la necesidad de mejorar la detección precoz — los ojos con muchas drusas o hiperpigmentación tienen mayor riesgo (21) - y la definición de protocolos de tratamiento que permitan mejorar la efectividad.

\section{AGRADECIMIENTOS}

Las autoras desean expresar su agradecimiento al Hospital del Oriente de Asturias por su colaboración en la recogida de datos y al Dr. Patricio Adúriz Alonso por desvelarnos algunos misterios de la oftalmología.

\section{BIBLIOGRAFÍA}

1. Congdon N, O'Colmain B, Klaver CC, Klein R, Munoz B, Friedman DS, et al. Causes and prevalence of visual impairment among adults in the United States. Arch Ophthalmol 2004; 122: 477-485.

2. Foran S, Wang JJ, Mitchell P. Causes of visual impairment in two older population cross-sections: the Blue Mountains Eye Study. Ophthalmic Epidemiol 2003; 10: 215-225.

3. Farber MD. National Registry for the Blind in Israel: estimation of prevalence and incidence rates and causes of blindness. Ophthalmic Epidemiol 2003; 10: 267-277.

4. Friedman DS, O'Colmain BJ, Munoz B, Tomany SC, McCarty C, de Jong PT, et al. Prevalence of age-related macular degeneration in the United States. Arch Ophthalmol 2004; 122: 564-572.

5. Jonasson F, Arnarsson A, Sasaki H, Peto T, Sasaki K, Bird AC. The prevalence of age-related maculopathy in Iceland: Reykjavik eye study. Arch Ophthalmol 2003; 121: 379-385.

6. Smith W, Assink J, Klein R, Mitchell P, Klaver CC, Klein $B E$, et al. Risk factors for age-related macular degeneration: Pooled findings from three continents. Ophthalmology 2001; 108: 697-704.

7. Oneill C, Jamison J, McCulloch D, Smith D. Age-related macular degeneration: cost-of-illnes issues. Drugs Aging 2001; 18: 233-241.

8. A prospective, randomized, double-masked trial on radiation therapy for neovascular age-related macular degeneration (RAD study). Radiation Therapy for Age-related Macular Degeneration. Ophthalmology 1999; 106: 2239-2247.

9. Marcus DM, Sheils W, Johnson MH, McIntosh SB, Leibach DB, Maguire A, et al. External beam irradiation of subfoveal choroidal neovascularization complicating agerelated macular degeneration: one-year results of a prospective, double-masked, randomized clinical trial. Arch Ophthalmol 2001; 119: 171-180.

10. Salinas Alamán A, García Layana A, Juberías Sánchez $J R$, Sánchez Tocino H, Sádaba Echarri LM, Moreno Montañes J. Termoterapia transpupilar en neovascularización subretiniana oculta secundaria a degeneración macular asociada a la edad. Primeros resultados. Arch Soc Esp Oftalmol 2002; 77: 617-622.

11. Oliva G. Terapia fotodinámica en el tratamiento de la degeneración macular asociada a la edad. Informe Técnico realizado por la Agència d'Avaluació de Tecnologia y Recerca Mèdiques. Barcelona: Agència d'Avaluació de Tecnologia y Recerca Mediques; 2002.

12. Verteporfin In Photodynamic Therapy Study Group. Verteporfin therapy of subfoveal choroidal neovascularization in age-related macular degeneration: two-year results of a randomized clinical trial including lesions with occult no classic choroidal neovascularization--verteporfin in photodynamic therapy report 2. Am J Ophthalmol 2001; 131: 541-560.

13. Bressler NM; Treatment of Age-Related Macular Degeneration with Photodynamic Therapy (TAP) Study Group. Photodynamic therapy of subfoveal choroidal neovascularization in age-related macular degeneration with verteporfin: two-year results of 2 randomized clinical trials-tap Report 2. Arch Ophthalmol 2001; 119: 198-207.

14. Rubin GS, Bressler NM. Treatment of Age-Related Macular Degeneration with Photodynamic therapy (TAP) study group. Effects of verteporfin therapy on contrast sensitivity: Results From the Treatment of Age-Related Macular Degeneration With Photodynamic Therapy (TAP) investigation-TAP report No 4. Retina 2002; 22: 536-544.

15. Brown GC, Sharma S, Brown MM, Kistler J. Utility values and age-related macular degeneration. Arch Ophthalmol 2000; 118: 47-51.

16. Aduriz-Lorenzo P, Ceballos M, Cienfuegos S, Díaz-Varela M, Natal-Ramos C, Muslera-Canclini E. Evaluación de un modelo de alta resolución para el tratamiento de la neovascularización subretiniana (NVSR) con verteporfín en el Principado de Asturias. Rev Calidad Asistencial 2003; 18: 405-467.

17. Sharma S, Brown GC, Brown MM, Hollands H, Shah GK The cost-effectiveness of photodynamic therapy for fellow eyes with subfoveal choroidal neovascularization secondary to age-related macular degeneration. Ophthalmology 2001; 108: 2051-2059.

18. Meads C, Hyde C. Photodynamic therapy with verteporfin is effective, but how big is its effect? Results of a sytematic review. Br J Ophthalmol 2004; 88: 212-217.

19. Sacristan JA, Oliva J, Del Llano J, Prieto L, Pinto JL. ¿Qué es una tecnología sanitaria eficiente en España? Gac Sanit 2002; 16: 334-343.

20. Hopley C. Salkeld G, Mitchell P. Cost utility of photodynamic therapy for predominantly classic neovascular age related macular degeneration. Br J Ophthalmol 2004; 88: 982-987.

21. Wang JJ, Foran S, Smith W, Mitchell P. Risk of age-related macular degeneration in eyes with macular drusen or hyperpigmentation: The Blue Mountains Eye Study cohort. Arch Ophthalmol 2003; 121 :658-663. 